\title{
Characteristics of Sleep-Conducive Music: A Narrative Evidence Review
}

\author{
Wang, Yuluan \\ MSc, Faculty of Rehabilitation Medicine, \\ University of Alberta \\ Rivard, Annette \\ $\mathrm{PhD}$, Occupational therapy research consultant \\ Department of Occupational Therapy, Faculty of Rehabilitation Medicine \\ University of Alberta. \\ Guptill, Christine \\ PhD and Assistant Professor \\ Department of Occupational Therapy, Faculty of Rehabilitation Medicine \\ University of Alberta \\ Boliek, Carol A. \\ Department of Communication Sciences and Disorders \\ Faculty of Rehabilitation Medicine, Neuroscience and Mental Health Institute \\ University of Alberta \\ Brown, Cary A. \\ PhD, Department of Occupational Therapy \\ Faculty of Rehabilitation Medicine \\ University of Alberta
}

\begin{abstract}
Objectives: Sleep deficiency (SD) is a prevalent problem and has serious negative consequences for physical, cognitive, and psychological well-being. The use of music as a non-pharmacological sleep intervention has been proposed in several studies. A 2014 meta-analysis of 10 randomized trials evaluating the impact of music on sleep concluded that it can decrease sleep onset delay (latency) and sleep disturbances, increases sleep duration, and improves daytime dysfunction. It appears that, to-date, evidence-based guidelines for the selection and/or production of sleeppromoting music do not exist. This review addresses that gap and synthesizes available literature towards the goal of developing guidelines grounded in the evidence-based characteristics of sleep conducive music.

Design and Results: A literature review of research papers relevant to the topic identified evidence-based characteristics of sleep-conducive music related to tempo, rhythm, pitch, volume, and duration.
\end{abstract}


Conclusion: This identification and compilation of evidence-based characteristics of sleepconducive music can underpin future research that targets development and testing of specific music to promote sleep.

Key Words: Sleep, Insomnia, Music, Sleep deficiency

\section{INTRODUCTION}

Sleep deficiency (SD) is a pervasive problem with significant negative outcomes for both physical and psychological health. According to the National Institutes of Health, SD occurs when sleep irregularity, insufficient sleep quality, or an untreated sleep disorder impairs optimal performance levels [1]. Due to the importance of sleep in physiological and psychological processes such as metabolic functions, neural detoxification, cognition, mood, and overall physical health, the role of sleep is critical to the enhancement of quality of life $[2,3]$. Research clearly demonstrates that SD increases an individual's risk of chronic conditions including diabetes, obesity, cardiovascular diseases, depression, and other mental health conditions [410]. Investigating ways to improve sleep quality is thus clearly important and, although the authors of two major reviews [11,12] found that the current evidence-base suffered a lack of methodological rigor, they concluded that the use of music as a non-pharmacological sleep intervention is low risk, cost effective, and holds much potential. One important gap in the foundational literature is a clear list of the components of music that contribute towards it being sleep conducive. The goal of this paper is to synthesise relevant research papers and generate an evidence-based guidelines for the components of sleep conducive music.

Music is defined as an intentional auditory stimulus with organized elements such as melody, rhythm, harmony, timbre, form, and style [13]. It is present in all cultures and is considered by some to be a low-cost health intervention with beneficial effects on physiological, psychological and socioemotional well-being [13]. Past research using electroencephalogram (EEG) measurement has shown that listening to sedative music can in fact lead to statistically significant increase in alpha wave activity [14-16]. Furman's early work further suggested that listening to music that promoted the rapid eye movement (REM) stage of sleep increased reports of happiness, while listening to music that promoted short wave sleep (SWS) increased boredom and sleepiness [14]. Beta wave neural activity, present in the REM stage of sleep, is associated with alertness; alpha wave activity is present in the SWS stage and is associated with relaxation and daydreaming; and theta and delta wave neural activity signify the non-REM light and deep stages of sleep that are associated with metabolism and other physiological functions, respectively [13]. More recent research, using EEGs in combination with actigraph analysis, confirmed that listening to sedative music while relaxing in bed can be associated with greater sleep efficiency (i.e., the percentage of time actually asleep in relation to the amount of time spent in bed), and promotes change from alpha to theta wave activity [17].

A 2017 survey at a large Canadian university found that 31.1\% of participating students used music to induce sleep [18]. The authors speculated that interventions involving music might be well received and hold relevance for both the concerning number of young adults with SD, and potentially, other populations. However, a preliminary literature search failed to retrieve a comprehensive, evidence-based list of the components required for a piece of music to be considered sleep-conducive. Consequently, this paper seeks to fill that gap and addresses the 
Wang, Y., Rivard, A., Guptill, C., Boliek, C. A. \& Brown, C. A. (2020) Characteristics of sleep-conducive Music: A narrative Evidence Review. Advances in Social Sciences Research Journal, 7(3) 431-437.

question, "what are the evidence-based criteria guiding selection and composition of sleepconducive music (SCM)"?

\section{Materials and Methods}

To address the current gap in the literature regarding identification of evidence-based music characteristics that are sleep-conducive we carried out a narrative review. The purpose of this project was to determine the existence of sufficient evidence to guide practice and inform future applied research, and was not intended to be a comprehensive, systematic review. With the assistance of a medical librarian, a comprehensive search of the literature was conducted (September-October 2018), seeking research studies that tested the influence of various music characteristics on sleep. Databases, including Scopus, Web of Science, and PubMed, were searched with the keywords "sleep deficiency, sleep music, music therapy, insomnia". We did not restrict our search to music-therapy literature as much of the relevant literature focused on neuro-physiological changes induced by different components of sound as tested with healthy participants. Papers were selected on the basis of their relevance to the topic, and studies that did not report research findings (e.g. opinion or theoretical papers) were excluded. Abstracts of articles that were retrieved in the database search were reviewed, and relevant material articles were retained for this paper. A summary of the findings is presented below.

\section{FINDINGS}

Our review of the literature revealed evidence that musical elements (such as sound, rhythm, melody, and harmony) may alter psychological and physiological responses by activating parasympathetic nervous system responses and alpha brain wave frequency to lower heart rate, respiratory rate, and blood pressure, and increase deep breathing and muscular relaxation. ${ }^{17,19}$ The use of sedative or relaxing music as an intervention has also been found to reduce activity in the neuroendocrine and sympathetic nervous system thereby reducing the human stress response through distraction from thoughts and decreased anxiety [13,20-22]. Finally, music has been found to reduce circulating noradrenaline, a neurotransmitter associated with awakening $[2,21,23]$. In addition to influencing neural networks, music listening has also been suggested to have a positive influence on hormone regulation. A study by Khalfa et al conducted with 24 post-secondary students, found that those who listened to relaxing music after engaging in a stressful task showed a decrease in salivary cortisol levels, whereas those who did not engage in music listening after the stress task showed continued elevated levels of salivary cortisol [24]. Additionally, patients undergoing surgery listening to music pre- and post-surgery for 30 minutes while on bed-rest showed a significant decrease in plasma cortisol levels [25], increased oxytocin levels, and increased subjective measures of relaxation [26]. Krout had earlier speculated that these effects might be due to the music's ability to obscure unwanted environmental stimuli such as noise, or its ability to provide distraction from unwanted stimuli such as stress or pain [27].

Whereas there is general agreement that music has potential physiological and psychological benefits that promote sleep, it is not appropriate to generalize these findings to all forms of music. As there are many genres of music, we cannot assume that any or all of them, or even that the entirety of any given genre (e.g., classical) will have the same sleep-conducive effect. 


\section{Music genres}

There are different genres or forms of music and each might be expected to have differing effects on individuals. Classical (Western Art) music emphasizes formal clarity, balance, and structure [28]. New age music, which includes designer music, is characterized as a less variable type of music with no central theme, no natural beat, and no recognizable melodies. It does, however, have repeated cycles of gentle undulating sounds that are meant to facilitate an environment for relaxation and meditation [28]. One seminal study measured reactions to classical, grunge rock, new age, and designer (created to enhance a sense of well-being) music [29]. Participants completed a survey examining tension (defined as a physiological reaction creating nervousness, edginess, and anxiety), mood, and mental clarity, both at baseline and immediately after listening to each kind of music. The results indicated that classical music decreased tension, sadness, and fatigue, but had little effect on other feelings such as relaxation and mental clarity. Listening to grunge rock was associated with increased feelings of hostility, fatigue, sadness, as well as increased levels of perceived tension along with significant feelings of decreased mental clarity, vigor, sense of compassion, and perceived relationship to others [29]. These findings were later supported by Chi and Young who reported that music with high pitches and faster tempos increased tension in participants [30]. Listening to new age music on the other hand, was associated with a significant increase in relaxation and mental clarity, and reductions in feelings of hostility and tension. After listening to designer music, which is created specifically to affect the listener on a physiological level, participants reported significantly more relaxation, daytime mental clarity and vigor, increased feeling of compassion, as well as decreased feelings of hostility, sadness, fatigue, and tension [30]. Wu et al determined, through study of participants' electroencephalogram (EEG) signals under exposure to different sounds, that REM stage activity increased with music that included fast rhythms, lively melodies, and a wide variety in note pitches [31]. In contrast, SWS stage EEG activity increased when the music encompassed low pitches and slower rhythms [31].

In summary, the available evidence suggests that music genre is not sufficient in itself to guide sleep-conducive music selection. Rather, a more nuanced examination of specific music elements is required. The following section identifies and describes the four distinct musical elements (Tempo, Rhythm and pitch, Volume, and Duration) that emerged from our review findings as have an evidence-based role in improving sleep. Interestingly, although timbre - the quality of musical sound, for e.g., what distinguishes the sound of a piano as compared to the violin - is a recognized as an essential characteristic element of music, we were unable to find any research articles regarding this element.

\section{Characteristics of sleep-conducive music}

\section{Tempo}

In musical terminology, tempo refers to the speed or pace of a composition. It is typically measured in beats per minute ( $\mathrm{bpm})$ and is not necessarily fixed in any given piece. Studies have demonstrated that music is sleep-conducive when it is without accented beats, percussive characteristics, or syncopation, and possesses a slow, steady tempo of 60-80 bpm (mimicking the human heart rate) and contains repetitive rhythms [32,33].

In a study with 58 post-secondary students, listening to classical music resulted in an average decrease in heart rate from $73 \mathrm{bpm}$ to $69.5 \mathrm{bpm}$, listening to new age music showed a decrease 
Wang, Y., Rivard, A., Guptill, C., Boliek, C. A. \& Brown, C. A. (2020) Characteristics of sleep-conducive Music: A narrative Evidence Review. Advances in Social Sciences Research Journal, 7(3) 431-437.

in heart rate from $73.45 \mathrm{bpm}$ to $68.8 \mathrm{bpm}$, and listening to popular music showed an increase in heart rate from $74 \mathrm{bpm}$ to $76 \mathrm{bpm} .{ }^{28} \mathrm{~A}$ review by Harris reported that listening to music with a tempo between 60 and 80 beats per minute (bpm) for 20 to 45 minutes before sleep was associated with faster sleep onset [34]. Similarly, a study with 24 young adults using polysomnography and self-reported analysis found that listening to what the researchers termed "sedating music" at $60 \mathrm{bpm}$ for one hour reduced sleep latency, and increased the duration of deep sleep stages, thus improving the quality of sleep [35].

\section{Rhythm and pitch}

The rhythm element in music refers to the placement of sounds (pitches) in time with a pattern of intervals between them. The characteristics of relaxing or sedative music are a slow, stable, and, sustained rhythm, along with low-frequency tones, soothing, and relaxing melodies, and low pitches [30]. A study by Morishima et al adjusted music and sound to participants' biological information (such as heartbeat and respiration rate) and found that a sustained sound was associated with shortened sleep latency (time elapsed from going to bed to falling asleep) compared to sounds that decayed and sounds that faded in and out [36].

\section{Volume}

Noise is defined as a set of unwanted sounds without control for elements such as volume (perceived loudness) and duration [37.38]. Noise exposure is a risk to physiological and psychological well-being, because of its influence on fatigue, insomnia, physiological stress and cardiovascular stimulations [38.39]. A noise level of 30 decibels (dB) or below on the other hand is considered to have no substantial biological effect on sleep [37-39]. Noise exposure above 30 $\mathrm{dB}$ has been found to prolong sleep latency, increase fragmented sleep, cause early awakenings, shallow sleep, heart rate and negative moods [40]. Noise levels between 30 to $40 \mathrm{dBs}$ are risk factors for arousal, awakenings, body movements, and sleep disturbances [41], and noise levels above $40 \mathrm{~dB}$ are a risk factor for sleep disturbances and delayed sleep onset [37.39.42]. Exposure to noise levels of $60 \mathrm{~dB}$ during sleep increases heart rate and physical movement throughout the night [40], and noise levels varying between 50 (road traffic) and $72 \mathrm{~dB}$ (aircraft) can decrease time spent in REM and SWS sleep stages [43]. Gradual exposure to noise between 40 to $70 \mathrm{~dB}$ appears likely to cause arousal during REM stage sleep. Moreover, certain types of noise, such as electric sounds and human voices cause an elevation in heart rate and increase the probability of arousal during the non-REM stage sleep compared to their impact on REM sleep [41]. In summary, the evidence supports that volume during music-listening to promote sleep should not exceed $30 \mathrm{~dB}$.

\section{Duration}

Harmat et al found that post-secondary students who listened to classical music for 45 minutes before sleep for a three-week duration showed better overall self-reported sleep quality from the second week onward [20]. A systematic review of 17 studies later confirmed that listening to music in bed, with closed eyes to minimize distractions, for 30-60 minutes per session is beneficial to perceived overall sleep quality [32]. Finally, a randomized controlled trial with 50 participants using polysomnography in addition to self-reported sleep quality, found that music listening for 45 minutes at bedtime for three consecutive days improved sleep quality by increasing duration of REM stage sleep and decreasing duration of stage 2 (light) sleep [44]. It appears that there is sufficient evidence to suggest that (a) duration of listening to music before 
sleep, (b) the number of consecutive days for listening, and (c) the number of weeks that music is employed, are all important variables to consider when developing treatment protocols.

\section{LIMITATIONS AND FUTURE RESEARCH}

It is important to note that the impact of music preference or prior musical training were not examined in the literature that was reviewed for this article. These may be important modifiers or confounders that should also be explored when researching the impact of music on sleep deficiency. For example, this review found that new age music may be conducive to sleep, due to its rhythmic, pitch and volume characteristics. However, if the person experiencing sleep deficiency strongly dislikes this genre of music, they might not achieve the desired physiological and psychological state conducive to sleep. Similarly, studies have demonstrated that musicians process music differently than non-musicians, which some evidence suggests has a measurable impact on the neurological structures of the brain. For example, musicians have increased grey matter volume in the motor, pre-motor, and somatosensory areas of the brain as compared to non-musicians [45]. The corpus callosum of musicians who begin training before age seven is larger in diameter than in non-musicians or musicians who began training later in life [46]. The implications of these differences for the use of music to improve sleep are not known. However, it seems plausible that professional musicians may attend to and process music differently (e.g. analytically; critically), and in ways that might alter its sleep conducive effects.

A final, but critical limitation to the development of comprehensive guidelines is that the research regarding the role of idiosyncratic music preference and psychological associations with certain songs and genres remains sparse. Encouragingly, foundational work in this area is emerging [11,47] and future guideline development will benefit from including criteria based on the intersections between physiology, psychology, and social aspects of music.

Whereas our review was thorough and aided by a medical librarian, it was not intended to serve as a systematic review. Therefore, the methodological quality of the studies reviewed has not been evaluated. At this time, it does not appear that sufficient high-quality studies exist in this line of inquiry to warrant such a review. Further examination of music variables, such as genre, speed (tempo), frequency (pitch), frequency variation, rhythmic structure, volume, and timbre, as well as non-music variables such as length of exposure, and their impact on sleep can help to elucidate music's utility as a sleep aid. The prevalence and significance of sleep deficiency warrant rigorous study of music as a non-pharmacological sleep intervention.

\section{IMPLICATIONS AND CONCLUSION}

As outlined in this article, sleep deficiency is a pervasive issue, which can have serious negative consequences for physical and psychological well-being across the life span. For example, postsecondary students may be particularly vulnerable to the impact of sleep deficiency and its consequences of increased risk for impaired cognition and mood disturbances, all highly problematic for academic achievement and social maturation. Other populations that may be vulnerable to sleep deficiency include older adults (including peri- and post-menopausal women), individuals with chronic neurological and musculoskeletal diseases, military members, and new parents. Given the accessibility and popularity of recorded music, it appears that music holds promise for use as a non-pharmacological intervention for sleep deficiency. However, there are currently no evidence-based, comprehensive guidelines for the selection and 
Wang, Y., Rivard, A., Guptill, C., Boliek, C. A. \& Brown, C. A. (2020) Characteristics of sleep-conducive Music: A narrative Evidence Review. Advances in Social Sciences Research Journal, 7(3) 431-437.

composition of sleep-conducive music. This review article addresses that gap and can serve as a foundation for primary research to further aid in the recognition and testing of musical elements that contribute to sleep and optimize music's sleep-conducive effects.

\section{Author Disclosure Statement}

No competing financial interests exist.

\section{Funding Information}

No funding was received for this article

\section{References}

National Institutes of Health: Sleep Deprivation and Deficiency. www.nhlbi.nih.gov/health-topics/sleep-deprivationand-deficiency (accessed 28 March 2018).

Huang CY, Chang ET, Lai HL. Comparing the effects of music and exercise with music for older adults with insomnia. Appl Nurs Res 2016;32:104-110.

Kadam YR, Patil SR, Waghachavare V, Gore AD. Influence of various lifestyle and psychosocial factors on sleep disturbances among the college students: a cross-sectional study from an urban area of India. JKIMSU 2016;53:51-60.

Agarwal A, Eryuzlu L, Chawla K. Sleep deprivation: student perceptions and its relationship to cardiovascular disease. Univ Toronto Med J 2015;92:34-38.

Chang SP, Shih KS, Chi CP et al. Association between exercise participation and quality of sleep and life among university students in Taiwan. Asia Pac J Public Health 2016;28:356-367.

Anothaisintawee T, Reutrakul S, van Cauter E, Thakkinstian A. Sleep disturbances compared to traditional risk factors for diabetes development: systematic review and meta-analysis. Sleep Med Rev 2016;30:11-24.

Van Cauter E, Spiegel K, Tasali E, Leproult R. Metabolic consequences of sleep and sleep loss. Sleep Med 2008;9:S23S28.

Huang W, Ramsey KM, Marcheva B, Bass J. Circadian rhythms, sleep, and metabolism. J Clin Invest 2011;121:21332141.

Kripke DF, Simons RN, Garfinkel L, Hammond EC. Short and long sleep and sleeping pills. Is increased mortality associated? Arch Gen Psychiatry 1979;36:103-16.

Wingard DL, Berkman LF. Mortality risk associated with sleeping patterns among adults. Sleep 1983;6:102-107.

Loewy J. Music Therapy as a Potential Intervention for Sleep Improvement. Nat Sci Sleep. 2020;12:1-9 https://doi.org/10.2147/NSS.S194938

Jespersen KV, Koenig J, Jennum P, Vuust P. Music for insomnia in adults. Cochrane Database Syst Rev. $2015 ; 8$. doi:10.1002/14651858.CD010459.pub

Kemper KJ, Danhauer SC. Music as Therapy. South Med J 2005;98:282-288.

12. Furman CE. The effect of musical stimuli on the brainwave production of children. J Music Ther 1978;15:108-117.

Verrusio W, Ettorre E, Vicenzini E et al. The Mozart effect: a quantitative EEG study. Conscious Cogn 2015;35:150-155.

Wagner MJ. Effect of music and biofeedback on alpha brainwave rhythms and attentiveness. J Res Music Educ 1975;23:3-13.

Phasukkit P, Mahrozeh N, Kumngern M, Tsungjotkusolmun S. A simple laboratory test of music therapy for insufficient sleep. In: $20158^{\text {th }}$ Biomedical Engineering International Conference, Pattaya, Thailand, 25-27 November 2015, pp, 15 .

Brown CA, Qin P, Esmail S. Sleep? Maybe later...A cross-campus survey of university students and sleep practices. Educ Sci 2017;7:66-81.

Solanki MS. Music: a non-invasive biological therapy or just a soothing melody? J Biomedical Sci 2016;5:30-33.

Harmat L, Takacs J, Bodizs R. Music improves sleep quality in students. J Adv Nurs 2008;62:327-335.

Lai HL, Good M. Music improves sleep quality in older adults. J Adv Nurs 2005;49:234-244. 
Tan LP. The effects of background music on quality of sleep in elementary school children. J Music Ther 2004;41:128150.

Grivel J, Cvetkovic V, Bayer $L$ et al. The wake-promoting hypocretin/orexin neurons change their response to noradrenaline after sleep deprivation. J Neurosci 2005;25:4127-4130.

Khalfa S, Bella SD, Roy $M$ et al. Effects of relaxing music on salivary cortisol level after psychological stress. Ann $N Y$ Acad Sci 2003;999:374-376

Nilsson U. Soothing music can increase oxytocin levels during bed rest after open-heart surgery: a randomised control trial. J Clin Nurs 2009;18:2153-2161.

Cervellin G, Lippi G. From music-beat to heart-beat: a journey in the complex interactions between music, brain and heart. Eur J Intern Med 2011;22:371-374.

Krout RE. Music listening to facilitate relaxation and promote wellness: integrated aspects of our neurophysiological responses to music. Arts Psychother 2007;34:134-141.

Mornhinweg GC. Effects of music preference and selection on stress reduction. J Holist Nurs 1992;10:101-109.

McCraty R. Barrios-Choplin B, Atkinson M, Tomasino D. The effects of different types of music on mood, tension and mental clarity. Altern Ther Health Med 1998;4:75-84.

Chi GC and Young A. Selection of music for inducing relaxation and alleviating pain. Holist Nurs Pract 2011;25:127135.

Wu D, Li CY, Yao DZ. Scale-free music of the brain. PLoS One 2009;4:e5915

Chan MF, Wong ZY, Thayala NV. The effectiveness of music listening in reducing depressive symptoms in adults: a systematic review. Complement Ther Med 2011;19:332-348.

Knight WE, Rickard NS. Relaxing music prevents stress-induced increases in subjective anxiety, systolic blood pressure, and heart rate in healthy males and females. J Music Ther 2011;38:254-272.

Harris MJ. Music as a transition for sleep. Occup Ther Now 2014;16:11-13

Chen $\mathrm{CK}$, Pei YC, Chen NH et al. Sedative music facilitates deep sleep in young adults. J Altern Complement Med 2014;20:312-317.

Morishima M, Sugino Y, Ueya Y et al. Effects on sleep by "cradle sound" adjusted to heartbeat and respiration. In: 2016 Advancement of Artificial Intelligence spring symposium series, Stanford, USA, 21-23 March 2016, pp, 387-393.

Muzet A. Environmental noise, sleep and health. Sleep Med Rev 2007;11:135-142.

Xie H, Kang J, Mills GH. Clinical review: the impact of noise on patients' sleep and the effectiveness of noise reduction strategies in intensive care units. Crit Care 2009;13:208-216.

Hume KI, Brink M, Basner M. Effects of environmental noise on sleep. Noise Health 2012;14:297-302.

Öhrström E, Björkman M. Effects of noise-disturbed sleep - a laboratory study on habituation and subjective noise sensitivity. J Sound Vib 1988;122:277-290.

Buxton OM, Ellenbogen JM, Wang W et al. Sleep disruption due to hospital noises: a prospective evaluation. Ann Intern Med 2012;157:170-180.

WHO night noise guidelines for Europe. World Health Organization. http://www.euro.who.int/en/healthtopics/environment-and-health/noise/policy/who-night-noise-guidelines-for-europe (accessed March 28 2018).

Griefahn B, Marks A, Robens S. Noise emitted from road, rail and air traffic and their effects on sleep. J Sound Vib 2006;295:129-140.

Chang E, Lai H, Chen P et al. The effects of music on the sleep quality of adults with chronic insomnia using evidence from polysomnographic and self-reported analysis: a randomized control trial. Int J Nurs Stud 2012;49:921-930.

Gaser C, Schlaug G. Brain structures differ between musicians and non-musicians. J Neurosci 2003;23:9240-9245.

Steele CJ, Bailey JA, Zatorre RJ, Penhune VB. Early musical training and white-matter plasticity in the corpus callosum: evidence for a sensitive period. J Neurosci 2013;33:1282-1290.

Trahan T, Durrant SJ, Müllensiefen D. Williamson VJ. The music that helps people sleep and the reasons they believe it works: a mixed methods analysis of online survey reports. PLoS One. 2018;13(11):e0206531. doi:10.1371/journal.pone.0206531 